\title{
Oridonin induces G2/M cell cycle arrest and apoptosis through MAPK and p53 signaling pathways in HepG2 cells
}

\author{
HUI WANG, YAN YE, JIAN-HONG CHU, GUO-YUAN ZHU, YING-WEI LI, \\ DAVID W.F. FONG and ZHI-LING YU \\ Center for Cancer and Inflammation Research, School of Chinese Medicine, Hong Kong Baptist University, \\ Kowloon Tong, Hong Kong, P.R. China
}

Received March 5, 2010; Accepted April 29, 2010

DOI: $10.3892 /$ or_00000903

\begin{abstract}
Oridonin, the main active constituent of Isodon rubescen, has antihepatocarcinoma activity in experimental and clinical settings. The aims of the study were to explore the anticancer effect of oridonin in HepG2 cells and to investigate the underlying mechanisms. Results showed that oridonin treatment for 24 or $48 \mathrm{~h}$ resulted in a marked decrease in cell viability time- and dose-dependently. $\mathrm{IC}_{50}$ values were determined as $38.86 \mu \mathrm{M}$ and $24.90 \mu \mathrm{M}$ for $24-\mathrm{h}$ and 48 -h treatments, respectively. Flow cytometric analysis showed that a $24-\mathrm{h}$ treatment of $40 \mu \mathrm{M}$ oridonin induced $\mathrm{G} 2 / \mathrm{M}$ cell cycle arrest and apoptosis. Typical apoptotic nucleus alterations were observed with fluorescence microscope after DAPI staining. Immunoblot analysis demonstrated that oridonin treatment increased expression levels of p-JNK, p-p38, p-p53 and p21, elevated the level of cyclin $\mathrm{B} 1 / \mathrm{p}-\mathrm{Cdc} 2$ (Tyr15) complex, and inhibited the expression of p-ERK. Moreover, oridonin treatment activated caspase-9 and caspase- 3 . In conclusion, oridonin induced G2/M cell cycle arrest and apoptosis in HepG2 cells through MAPK and p53 pathways, which advances our understanding on the molecular mechanisms of oridonin in hepatocarcinoma management.
\end{abstract}

\section{Introduction}

Hepatocellular carcinoma (HCC) is among the most lethal and prevalent cancers in the human population. Despite its significance, there are only limited therapeutic options (1). Traditional Chinese medicine (TCM) can provide new avenues for alternative treatments of HCC (2). Chinese medicinal herb Isodon rubescens (Hemsl.) C.Y. Wu et Hsuan, traditionally used for the management of respiratory and gastrointestinal bacterial infections, inflammation, and cancer, has been shown to be effective in HCC management (3). Oridonin

Correspondence to: Dr Zhi-Ling Yu, Center for Cancer and Inflammation Research, School of Chinese Medicine, Hong Kong Baptist University, Kowloon Tong, Hong Kong, P.R. China E-mail: zlyu@hkbu.edu.hk

Key words: apoptosis, G2/M arrest, HepG2, MAPK, oridonin, p53 is the main active constituent of I. Rubescens and also showed curative effect in liver carcinoma patients (4). In vitro experiments showed that oridonin could inhibit the growth of human hepatocellular carcinoma BEL-7402 cells and cause apoptosis via the inhibition of telomerase activity (5). Oridonin can also induce apoptosis through the generation of reactive oxygen species (ROS) in human hepatoma HepG2 cells (6). Whether other mechanisms are involved in the anti-HCC activity of oridonin remains to be addressed.

The mitogen-activated protein kinases (MAPKs) including the excellular signal-regulated kinase (ERK1/2), c-Jun Nterminal kinase (JNK), and p38, are suggested to play important roles in cell proliferation and apoptosis, which have been implicated in various cancers including HCC $(7,8)$. MAPKs have been shown to phophorylate p53 in response to different stressful stimuli, and such phosphorylation can initiate the p53 response, leading to cell cycle arrest and apoptosis (9). In the present study, we investigated the involvement of MAPK and p53 signaling pathways in the anticancer effects of oridonin in HepG2 cells.

\section{Materials and methods}

Reagents and antibodies. Oridonin was purchased from Shanghai Shamrock Imp and Exp Trading Co., Ltd., and the purity was determined to be $97 \%$ by HPLC. MTT (3-(4,5dimethylthiazol-2-yl)-2,5-diphenyl tetrazolium bromide) was from USB Corporation. Ribonuclease A (RNase A), propidium iodide (PI), and 4,6-diamidino-2-phenylindole (DAPI) were from Sigma-Aldrich Biotechnology. Annexin V-FITC Apoptosis Detection Kit I was obtained from BD Bioscience. Protease inhibitor cocktail and phosphatase inhibitor cocktail were from Roche Bioscience. Bio-Rad Protein Assay was from Bio-Rad Laboratories, Inc. Nitrocellulose membrane and ECL detection reagents were from Amersham Biosciences. ERK, p-ERK (Thr202/Tyr204), JNK, p-JNK (Thr183/Tyr185), p38, p-p38 (Thr180/Tyr182), p-p53 (Ser15), p21, p-Cdc2 (Tyr15), and cleaved-caspase-3 antibodies were from Cell Signaling Technology; caspase-3, caspase-9, B-actin, and anti-rabbit IgG antibodies (horseradish peroxidase conjugated) were from Santa Cruz Biotechnology; cyclin-B1 antibody was from Upstate Biotechnology; anti-mouse IgG antibody (horseradish peroxidase conjugated) was from Invitrogen Biotechnology. 
Cell culture and drug treatment. HepG2 cells (ATCC, USA), grown in Dulbecco's modified Eagle's medium (DMEM, Gibco, USA) supplemented with $10 \%$ fetal bovine serum (FBS, Gibco) and 1\% penicillin/streptomycin (P/S, Gibco) were cultured at $37^{\circ} \mathrm{C}$ in an atmosphere containing $5 \% \mathrm{CO}_{2}$.

Stock solution of oridonin $(274.5 \mathrm{mM})$ was prepared in dimethyl sulfoxide (DMSO, Sigma, France). Aliquots were stored at $-20^{\circ} \mathrm{C}$. HepG2 cells were treated with various concentrations of oridonin for indicated durations. Control cells were treated with $0.1 \%$ DMSO.

Cell viability assay. HepG2 cells $\left(5.0 \times 10^{3}\right.$ cells per well $)$ were seeded and grown in 96-well plates for $24 \mathrm{~h}$ and then treated with various concentrations of oridonin. After a 24or 48 -h incubation, $10 \mu \mathrm{l}$ of $5 \mathrm{mg} / \mathrm{ml}$ MTT solution was added to each well and plates were incubated at $37^{\circ} \mathrm{C}$ for $4 \mathrm{~h}$. Following medium removal, $100 \mu 1$ of DMSO was added to each well and plates were gently shaken for $5 \mathrm{~min}$. Optical absorbance was determined at $570 \mathrm{~nm}$ with a microplate spectrophotometer (BD Bioscience, USA). Absorbance obtained by vehicle-treated cells was rated as $100 \%$ of cell survival. Each treatment was performed in triplicate and each experiment was repeated three times.

Flow cytometric analyses of cell cycle distribution and apoptosis. Cells were seeded at a density of $60 \times 10^{4}$ per $60-\mathrm{mm}$ dish and grown overnight. Oridonin was added to a final concentration of $40 \mu \mathrm{M}$ and cells were incubated for $24 \mathrm{~h}$. Both detached and adherent cells were collected and centrifuged at $1000 \mathrm{~g}$ for $5 \mathrm{~min}$ at $4^{\circ} \mathrm{C}$. Pellets were rinsed with ice-cold phosphate-buffered saline (PBS) and fixed with $70 \%$ ethanol for $2 \mathrm{~h}$. Cells were then stained with staining buffer (PBS containing $20 \mu \mathrm{g} / \mathrm{ml}$ of PI, $100 \mu \mathrm{g} / \mathrm{ml}$ RNase A, and $0.1 \%$ Triton $\mathrm{X}-100$ ) for $15 \mathrm{~min}$ at $37^{\circ} \mathrm{C}$ in the dark. Samples were analyzed by a flow cytometer (BD Bioscience).

Early and late phase apoptotic cells were monitored with Annexin V-FITC Apoptosis Detection Kit I. Cells were washed twice with cold PBS, resuspended in binding buffer, and incubated with FITC and PI staining solution following manufacturer's instructions. Samples of 10,000 stained cells were analyzed by flow cytometry.

Apoptotic morphology observation. Apoptotic morphology was monitored in DAPI stained cells. Cells $\left(40 \times 10^{4}\right)$ were grown for $24 \mathrm{~h}$ on cover slips in $35-\mathrm{mm}$ dishes in the presence or absence of $40 \mu \mathrm{M}$ oridonin. Cover slips were carefully washed with PBS, fixed with $4 \%$ paraformaldehyde for $10 \mathrm{~min}$ and incubated with $10 \mu \mathrm{g} / \mathrm{ml}$ DAPI for $10 \mathrm{~min}$. Cells were washed with PBS and observed under a fluorescent microscope (Nikon, Japan).

Western blot analysis. Cells treated with $40 \mu \mathrm{M}$ oridonin for indicated durations were collected and proteins were extracted with RIPA lysis buffer [50 mM Tris-Cl, $1 \% \mathrm{v} / \mathrm{v}$ $\mathrm{NP}-40,0.35 \% \mathrm{w} / \mathrm{v}$ sodium-deoxycholate, $150 \mathrm{mM} \mathrm{NaCl}$, 1 mM EDTA, 1 mM EGTA, pH 7.4, 1 mM phenylmethylsulfonyl fluoride (PMSF), $\left.1 \mathrm{mM} \mathrm{NaF}, 1 \mathrm{mM} \mathrm{Na} \mathrm{VO}_{4}\right]$ containing a protease inhibitor cocktail and a phosphatase inhibitor cocktail. Protein concentration was determined by Bio-Rad Protein Assay. The individual protein samples

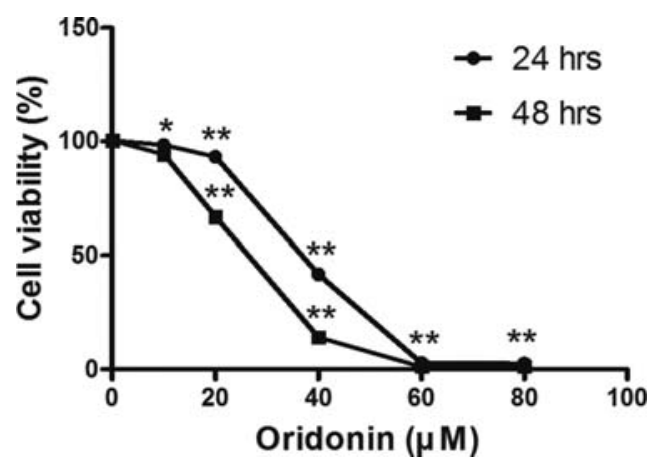

Figure 1. Oridonin inhibited HepG2 cell proliferation. Cells were treated with various consentrations of oridonin or vehicle for 24 or $48 \mathrm{~h}$. Cell viability was determined by MTT assay. Data are expressed as mean \pm SEM from three independent experiments. ${ }^{*} \mathrm{p}<0.05,{ }^{* *} \mathrm{p}<0.01$ vs. the control.
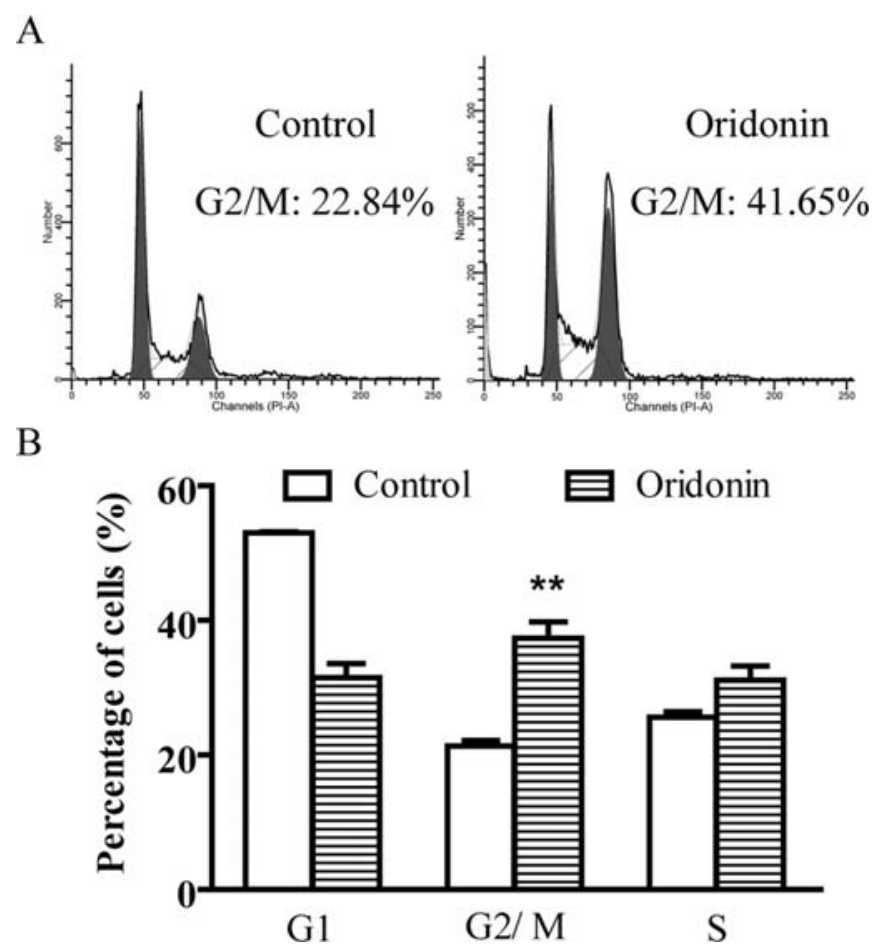

Figure 2. Oridonin induced cell cycle arrest in HepG2 cells. Cells were treated with $40 \mu \mathrm{M}$ oridonin or vehicle for $24 \mathrm{~h}$ and stained with PI. Cellular DNA contents were monitored by flow cytometry. (A) Results are representative of three independent experiments. (B) Cell cycle distribution determined using Modfit software version 3.1. The proportions of G1, S and $\mathrm{G} 2 / \mathrm{M}$ phase cells are shown as indicated and presented as the mean $\pm \mathrm{SEM}$ of three independent experiments. ${ }^{* *} \mathrm{p}<0.01 \mathrm{vs}$ the control.

(20-50 $\mu \mathrm{g}$ ) were separated by SDS-PAGE and then electrotransferred onto the nitrocellulose membrane. Membranes were blocked for 30 min with 3\% skim-milk in TBST buffer composed of $50 \mathrm{mM}$ Tris (pH 7.6), $150 \mathrm{mM}$ $\mathrm{NaCl}$ and $0.1 \%$ Tween-20 and incubated with primary antibodies overnight at $4{ }^{\circ} \mathrm{C}$ followed by incubation with horseradish peroxidase-conjugated secondary antibodies. Protein signals were visualized by ECL detection reagents according to manufacturer's instruction. 
A

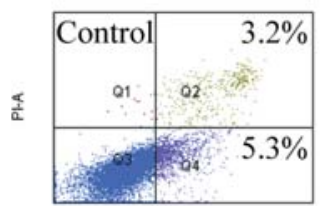

FITC-A

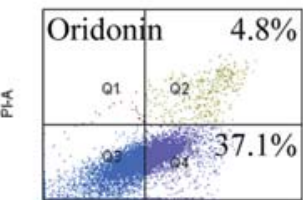

FITC.A

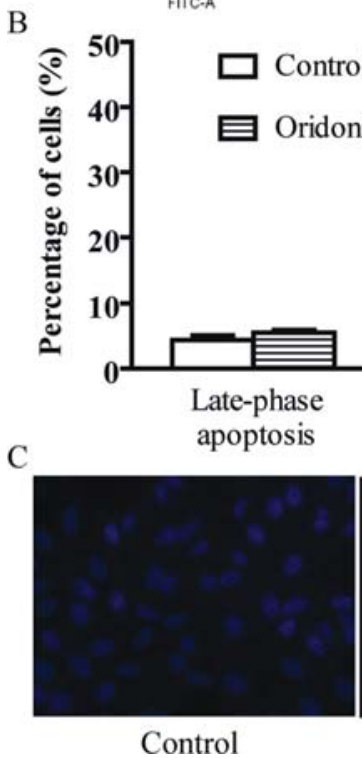

Control

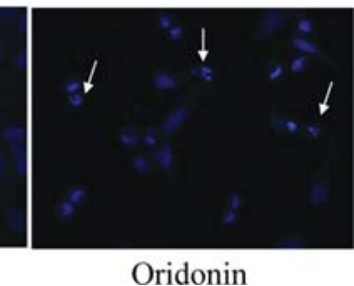

Oridonin
Figure 3. Oridonin induced apoptosis in HepG2 cells. Cells were treated with $40 \mu \mathrm{M}$ oridonin or vehicle for $24 \mathrm{~h}$. (A) HepG2 cells were stained with Annexin V-FITC/PI and analyzed by flow cytometry. Results are representative of three independent experiments. (B) The percentage of apoptotic cells is presented as the mean \pm SEM of three independent experiments. ${ }^{* *} \mathrm{p}<0.01$ vs the control. (C) Oridonin-treated and -untreated cells were stained with DAPI and visualized by fluorescent microscopy (x400). Representative DAPI-stained nuclei of cells are shown. Typical apoptotic changes of nucleus (chromatin condensation, nuclear fragmentation, appearance of apoptotic bodies) are indicated by arrows.

Data analysis. All results are expressed as the mean \pm SEM and statistical analyses were performed using the Student's t-test.

\section{Results}

Cytotoxicity of oridonin. MTT assay showed that treatment with oridonin for 24 or $48 \mathrm{~h}$ resulted in a marked decrease in cell viability in a time- and dose-dependent manner (Fig. 1). The $\mathrm{IC}_{50}$ values were determined as $38.86 \mu \mathrm{M}$ for $24-\mathrm{h}$ and $24.90 \mu \mathrm{M}$ for 48 -h treatments, respectively, from the doseresponse curve with GraphPad Prism 5.0 software (GraphPad software, USA). In the following assays $40 \mu \mathrm{M}$ oridonin was used.

Cell cycle arrest and apoptosis induced by oridonin. To verify whether oridonin caused cell cycle perturbation, DNA contents of HepG2 cells treated for $24 \mathrm{~h}$ with or without $40 \mu \mathrm{M}$ oridonin were analyzed using a flow cytometer equipped with the Modfit software version 3.1 (Verity Software House, USA). Fig. 2A showed that oridonin treatment caused a significant increase of $\mathrm{G} 2 / \mathrm{M}$ phase cells. The percentage of $\mathrm{G} 2 / \mathrm{M}$ phase cells increased from

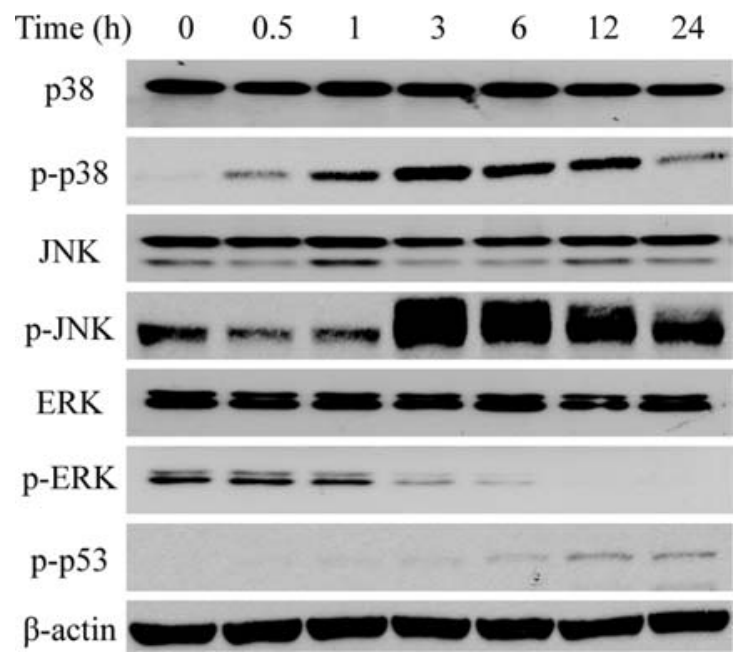

Figure 4. Time-dependent effects of oridonin on protein expression levels of ERK, p-ERK, JNK, p-JNK, p38, p-p38 and p-p53. The protein expression in HepG 2 cells treated with $40 \mu \mathrm{M}$ oridonin from 0 to $24 \mathrm{~h}$ was analyzed by Western blotting. ß-actin was included as a protein-loading control. Results shown are the representative of three independent experiments.

$21.34 \pm 0.82 \%$ to $37.38 \pm 2.37 \%$ after oridonin treatment (Fig. 2B).

To investigate the occurrence of apoptosis, flow cytometric analysis was performed using Annexin V-FITC/PIstained HepG2 cells. Results showed that oridonin treatment for $24 \mathrm{~h}$ significantly induced early phase apoptosis but had no effect on late phase apoptosis (Fig. 3A and B). Fig. 3A shows representative results of 3 independent experiments. Early-phase apoptosis rate was $34.37 \pm 4.16 \%$ in oridonintreated cells vs. $4.20 \pm 0.70 \%$ in control cells (Fig. 3B). The morphology changes of apoptotic cells were observed under a fluorescence microscope after DAPI staining. Typical apoptotic nucleus alterations (chromatin condensation, nuclear fragmentation, appearance of apoptotic bodies) were observed after oridonin treatment (Fig. 3C).

Oridonin increased p-JNK, p-p38, p-p53 and decreased p-ERK protein expression levels. To determine whether the MAPKs cascade and their downstream p53 protein are involved in the anti-cancer effects of oridonin, Western blot analysis was applied. As shown in Fig. 4, the expression levels of ERK, JNK and p38 proteins were not affected by oridonin. However, treatment of oridonin significantly and persistently increased the expression levels of phosphorylated p38 (p-p38) and $\mathrm{p}-\mathrm{JNK}$, and decreased the expression level of p-ERK. Oridonin treatment also up-regulated p-p53 in a time-dependent manner.

Oridonin increased the expression of $p 21$ and the inactive form of cyclin B1/Cdc2 complex. We investigated the effect of oridonin on molecules involved in cell cycle progression including p21, cyclin-B1 and p-Cdc2. As shown in Fig. 5, oridonin treatment for $24 \mathrm{~h}$ resulted in an up-regulation of $\mathrm{p} 21$ in HepG2 cells. In addition, oridonin treatment elevated the level of cyclin B1/p-Cdc2 (Tyr15), which is an inactive form of the cyclin B1/Cdc2 complex and a hallmark for $\mathrm{G} 2 / \mathrm{M}$ accumulation or mitotic delay (10). 


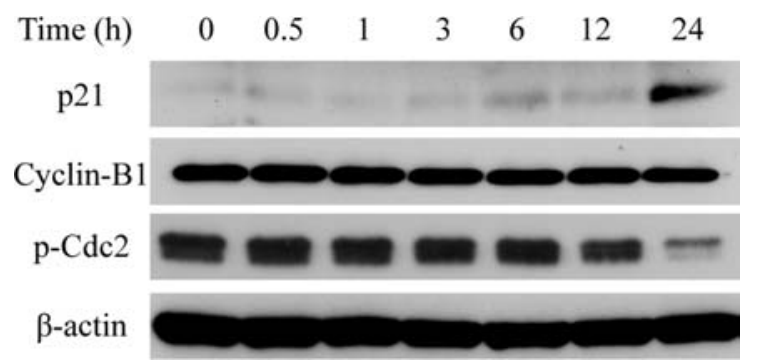

Figure 5. Time-dependent effects of oridonin on the protein expression levels of p21, cyclin-B1 and p-Cdc2. The protein expression in HepG2 cells treated with $40 \mu \mathrm{M}$ oridonin from 0 to $24 \mathrm{~h}$ was analyzed by Western blotting. B-actin was included as a protein-loading control. Results shown are representative of three independent experiments.

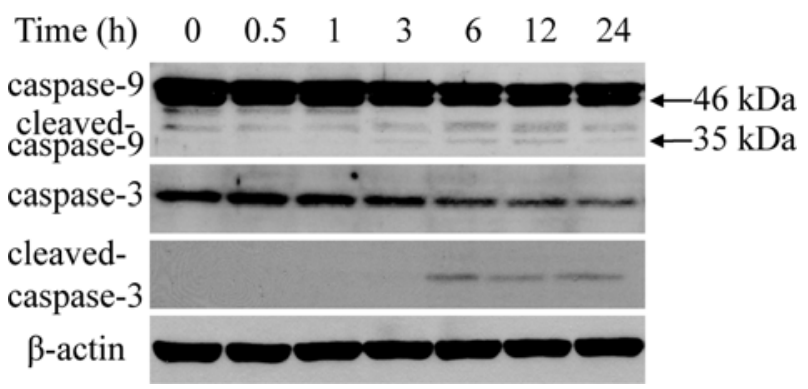

Figure 6. Time-dependent effects of oridonin on the protein expression levels of caspase- 9 , cleaved caspase- 9 , caspase- 3 and cleaved caspase-3. Protein expression in HepG2 cells treated with $40 \mu \mathrm{M}$ oridonin from 0 to $24 \mathrm{~h}$ was analyzed by Western blotting. $\beta$-actin was included as a proteinloading control. Results shown are representative of three independent experiments.

Oridonin activated caspase cascades. We next investigated the involvement of caspase cascades in oridonin-induced apoptosis by immunoblotting. As shown in Fig. 6, oridonin treatment decreased the expression of caspase- 9 (46 kDa) and caspase- 3 , as well as activated the cleavage of caspase- 9 and caspase- 3 evidenced by the increased expression levels of cleaved caspase-9 (35 kDa) and cleaved caspase-3.

\section{Discussion}

Previous studies have shown that oridonin has anti-hepatocarcinoma activities in vivo and in vitro (4-6). Here, we demonstrated that oridonin inhibited proliferation of HepG2 cells by arresting the cells in the G2/M phase and inducing apoptosis. Western blot analysis showed that p53 and MAPKs including ERK, p38 and JNK were involved in the effects of oridonin. The proposed action mechanisms of oridonin in HepG2 cells are summarized in Fig. 7.

MAPKs play different biological roles cell type and treating agent dependently in various experimental settings (11). Oridonin has been demonstrated to be able to cause ERK activation $(12-15)$ and p38 inactivation $(13,15)$ in human macrophage-like U937 cells, murine fibrosarcoma L929 cells and human melanoma A375-S2 cells without affecting JNK activity $(12,13,15)$. Other reports claimed that oridonin could inactivate JNK (15) in L929 cells, and inactivate ERK $(11,16)$

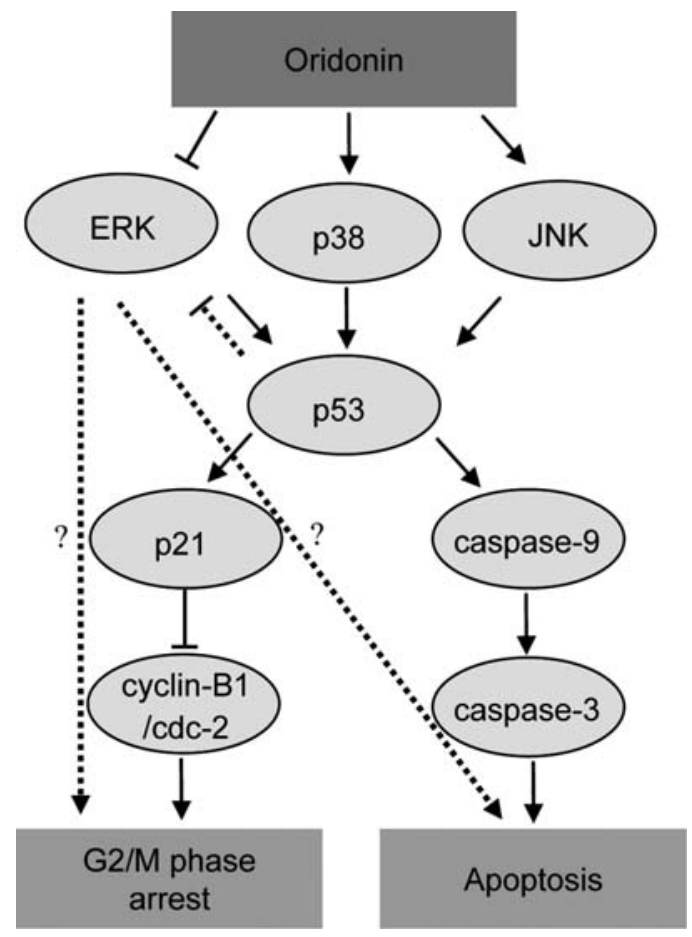

Figure 7. Proposed mechanisms for the G2/M arrest and apoptosis induced by oridonin in HepG 2 cells. Dotted lines represent mechanisms not investigated in this study. $\downarrow$ stimulation; $\perp$, inhibition.

but activate p38 and JNK (11) in human osteosarcoma cells and epidermoid carcinoma A431 cells. P38 and JNK function as upstream kinases of p53 phosphorylation and subsequently stabilize and activate p53 transcriptional activity, leading to diverse cellular responses, such as cell cycle arrest and apoptosis (9). We found that in response to oridonin treatment increased expression of p-p38 and p-JNK was accompanied by the up-regualtion of $\mathrm{p}-\mathrm{p} 53$, which suggests that p38 and JNK activation are involved in the G2/M arresting and apoptotic activities of oridonin in HepG2 cells. Consistent with our observations, a p38 inhibitor has been shown to be able to partially inhibit cell death and the activation of p53 in oridonin-treated HepG2 cells (6). Unlike p38 and JNK, ERK serves as a protective mechanism in cell cycle arrest and apoptosis in some cell types. Although phosphorylation of $\mathrm{p} 53$ by ERK was observed in a number of experimental systems (9), ERK has been reported to be inactivated by p53-regulated transcription of phosphatases MKP1, PAC1 and DUSP5 (9). In human HCC cell lines, multiple anticancer effects such as inhibition of cellular proliferation as well as induction of cell cycle arrest and apoptosis have been achieved by blocking ERK signalling (17). ERK inactivation observed in this study may contribute to the G2/M cell cycle arresting and apoptotic activities of oridonin, which needs to be further investigated.

Malfunction of p53 has been observed in up to $50 \%$ of HCC (17). P53 is implicated in the induction of both growth arrest and apoptosis. In response to stimuli, p53 increases the transcription of $\mathrm{p} 21$, which directly binds to and inhibits the activity of the cyclin B1/Cdc2 complex thus cause G2/M phase cell cycle arrest (18). After oridonin treatment for $24 \mathrm{~h}$, we found that increased phosphorylation of p53 is 
accompanied by the increased expression of $\mathrm{p} 21$ and elevated ratio of cyclin-B1/p-Cdc2, an inactive form of the cyclin B1/ $\mathrm{Cdc} 2$ complex and a hallmark for $\mathrm{G} 2 / \mathrm{M}$ accumulation or mitotic delay (10). These observations suggest that p53 and p21 are involved in oridonin-induced G2/M cell cycle arrest. In agreement with our findings a p53 inhibitor pifithrin has been shown to be able to significantly reduce oridonin-induced apoptosis in HepG2 cells (6). The activation of caspase-9 and caspase-3 is required for the p53-dependent apoptosis induction (19). In our experimental conditions, protein expression of cleaved caspase- 9 and caspase- 3 were increased in response to oridonin challenge, suggesting that oridonin-induced apoptosis is mediated, at least in part, by p53 through the activation of caspase cascade in HepG2 cells.

In conclusion, we found that oridonin induced G2/M cell cycle arrest and apoptosis in HepG2 cells through MAPK and p53 pathways. G2/M cell cycle arrest in HepG2 cells was observed for the first time in this study. Findings of this study advance our understanding on the molecular mechanisms of oridonin in HCC intervention.

\section{Acknowledgements}

This study was supported by a research grant (FRG/08-09/I08) from Hong Kong Baptist University.

\section{References}

1. Farazi PA and DePinho RA: Hepatocellular carcinoma pathogenesis: from genes to environment. Nat Rev Cancer 6: 674-687, 2006.

2. Luk JM, Wang X, Liu P, et al: Traditional Chinese herbal medicines for treatment of liver fibrosis and cancer: from laboratory discovery to clinical evaluation. Liver Int 27: 879-890, 2007.

3. Sun HD, Huang SX and Han QB: Diterpenoids from isodon species and their biological activities. Nat Prod Rep 23: 673-698, 2006.

4. Wang RL: Dong ling cao zhi liao yuan fa xing gan ai 31 li lin chuang guan cha. Ai Zheng 3: 50, 1984.

5. Zhang JF, Chen GH, Lu MQ and Liu JJ: Antiproliferation effects of oridonin on hepatocellular carcinoma BEL-7402 cells and its mechanism. Chinese Traditional Patent Medicine 28: 1325-1329, 2006.
6. Huang J, Wu L, Tashiro S, Onodera S and Ikejima T: Reactive oxygen species mediate oridonin-induced HepG2 apoptosis through p53, MAPK, and mitochondrial signaling pathways. J Pharmacol Sci 107: 370-379, 2008

7. Widmann C, Gibson S, Jarpe MB and Johnson GL: Mitogenactivated protein kinase: conservation of a three-kinase module from yeast to human. Physiol Rev 79: 143-180, 1999.

8. Wiesenauer CA, Yip-Schneider MT, Wang Y and Schmidt CM: Multiple anticancer effects of blocking MEK-ERK signaling in hepatocellular carcinoma. J Am Coll Surg 198: 410-421, 2004.

9. Wu GS: The functional interactions between the p53 and MAPK signaling pathways. Cancer Biol Ther 3: 156-161, 2004.

10. Yan L, Donze JR and Liu L: Inactivated MGMT by O6-benzylguanine is associated with prolonged $\mathrm{G} 2 / \mathrm{M}$ arrest in cancer cells treated with BCNU. Oncogene 24: 2175-2183, 2005.

11. Jin S, Shen JN, Wang J, Huang G and Zhou JG: Oridonin induced apoptosis through AKT and MAPKS signaling pathways in human osteosarcoma cells. Cancer Biol Ther 6: 261-268, 2007.

12. Liu YQ, You S, Tashiro S, Onodera S and Ikejima T: Activation of phosphoinositide 3-kinase, protein kinase c, and extracellular signal-regulated kinase is required for oridonin-enhanced phagocytosis of apoptotic bodies in human macrophage-like U937 cells. J Pharmacol Sci 98: 361-371, 2005.

13. Zhang CL, Wu LJ, Zuo HJ, Tashiro S, Onodera S and Ikejima T: Cytochrome c release from oridonin-treated apoptotic A375-S2 cells is dependent on p53 and extracellular signal-regulated kinase activation. J Pharmacol Sci 96: 155-163, 2004

14. Liu YQ, Mu ZQ, You S, Tashiro S, Onodera S and Ikejima T: Fas/FasL signaling allows extracelluar-signal regulated kinase to regulate cytochrome $\mathrm{c}$ release in oridonin-induced apoptotic U937 cells. Biol Pharm Bull 29: 1873-1879, 2006.

15. Zhang CL, Wu LJ, Tashiro S, Onodera S and Ikejima T: Oridonin induces a caspase-independent but mitochondriaand MAPK-dependent cell death in the murine fibrosarcoma cell line L929. Biol Pharm Bull 27: 1527-1531, 2004.

16. Li D, Wu LJ, Tashiro S, Onodera S and Ikejima T: Oridonininduced A431 cell apoptosis partially through blockage of the RAS/RAF/ERK signal pathway. J Pharmacol Sci 103: 56-66, 2007.

17. Chaparro M, Gonzalez Moreno L, Trapero-Marugan M, Medina J and Moreno-Otero R: Review article: pharmacological therapy for hepatocellular carcinoma with sorafenib and other oral agents. Aliment Pharmacol Ther 28: 1269-1277, 2008.

18. Cheng Y, Qiu F, Ye YC, Tashiro S, Onodera S and Ikejima T: Oridonin induces G2/M arrest and apoptosis via activating ERK-p53 apoptotic pathway and inhibiting PTK-RAS-RAFJNK survival pathway in murine fibrosarcoma L929 cells. Arch Biochem Biophys 490: 70-75, 2009.

19. Shen Y and White E: P53-dependent apoptosis pathways. Adv Cancer Res 82: 55-84, 2001. 